\title{
Linear Carbon Chains inside Multi-Walled Carbon Nanotubes: Growth Mechanism, Thermal Stability and Electrical Properties
}

Cheon-Soo Kang ${ }^{1}$, Kazunori Fujisawa ${ }^{1,4}$, Yong-Il Ko ${ }^{1}$, Hiroyuki Muramatsu ${ }^{1}$, Takuya Hayashi $^{1, *}$, Morinobu Endo ${ }^{1}$, Hee Jou Kim², Daun Lim $^{2}$, Jin Hee Kim ${ }^{3}$, Yong Chae Jung ${ }^{4}$, Mauricio Terrones ${ }^{1,5}$, Mildred S. Dresselhaus ${ }^{6}$, Yoong Ahm Kim ${ }^{1,2, *}$

${ }^{1}$ Institute of Carbon Science and Technology \& Faculty of Engineering, Shinshu University, 4-17-1 Wakasato, Nagano 380-8553, Japan

${ }^{2}$ Alan G. MacDiarmid Energy Research Institute, School of Polymer Science and Engineering \& Department of Polymer Engineering, Graduate School, Chonnam National University, 77 Yongbong-ro, Buk-gu, Gwangju 500-7257, Republic of Korea

${ }^{3}$ Biohousing Research Institute, Chonnam National University, 77 Yongbong-ro, Buk-gu, Gwangju 500-7257, Republic of Korea

${ }^{4}$ Institute of Advanced Composite Materials, Korea Institute of Science and Technology (KIST), Chudong-ro 92, Bongdong-eup, Wanju-gun, Jeonbuk 565-905, Republic of Korea

${ }^{5}$ Department of Physics, Department of Chemistry, Department of Materials Science \& Engineering, and Center for 2-Dimensional and Layered Materials, The Pennsylvania State University, University Park, PA 16802, USA

${ }^{6}$ Department of Electrical Engineering and Computer Science, Department of Physics, Massachusetts Institute of Technology, Cambridge, Massachusetts 02139-4307, USA

\begin{abstract}
Linear carbon chains (LCCs) consisting of $s p$-hybridized carbon atoms are considered a fascinating 1D system and could be used in the fabrication of the next-generation molecular devices because of its ideal linear atomic nature. A large portion of long LCCs inside multi-walled carbon nanotubes (MWCNTs) were synthesized by atmospheric arc discharge in the presence of boron. Closed-end growth of MWCNTs in the arc process is suggested as a critical condition for the simultaneous growth of LCCs within the inner cores of carbon nanotubes. The strong Raman line around $1850 \mathrm{~cm}^{-1}$ was used to characterize the degree of
\end{abstract}


filling as well as their structural stability under high temperature thermal treatments. We observed a distinctive change in the electrical conductivity of the MWCNT assembly before and after the disappearance of LCCs due to the expected strong coupling interaction between the LCCs and the innermost tube. This work demonstrates for the first time the enhanced effect of confined linear carbon chains on the overall electrical conductivity of MWCNT assemblies.

Keywords: Linear carbon chain, Multi-walled carbon nanotube, Electrical conductivity, Thermal stability, Charge transfer

*Corresponding authors.

E-mail addresses: hayashi@endomoribu.shinshu-u.ac.jp (T. Hayashi), yak@jnu.ac.kr (Y.A. Kim)

\section{Introduction}

In recent years, linear carbon chains (LCCs) consisting of $s p$-hybridized carbon atoms attracted the attention of many researchers mainly because of their ideal 1D nature at the atomic scale $[1,2]$. Detailed theoretical studies on LCCs proposed stronger mechanical strength than diamond and higher electron mobility when compared to graphene and carbon nanotubes [3, 4]. Geometrically, LCCs are expected to have two different configurations of carbon atoms; one is an alternating triple and single bonds (called polyyne, $-\mathrm{C} \equiv \mathrm{C}-\mathrm{C} \equiv \mathrm{C}-\mathrm{C} \equiv \mathrm{C}-$ ) and the other is sequential double bonds (called cumulene, $=\mathrm{C}=\mathrm{C}=$ $\mathrm{C}=\mathrm{C}=\mathrm{C}=\mathrm{C}=$ ). The former is expected to have controllable bandgap whereas the latter should show superconducting behavior. The electronic and optical properties of LCCs can be modified by altering the number of carbon atoms as well as the type of terminal groups. Such theoretically predicted properties will allow LCCs to be used in the next-generation electronic devices, such as molecular wires, and optical and energy storage devices [5-8].

From the viewpoint of experimental synthesis, LCCs can be grown both physically, through sputtering and chemical vapor deposition (CVD), and chemically by detaching hydrogen atoms from acetylene derivatives. In addition, LCCs have been synthesized by 
directing an electron beam onto few layered graphene or carbon nanotubes, inside a transmission electron microscope (TEM) [9-11]. Two groups reported the effective growth of short polyynes using arc discharge or laser ablation in the liquid phase [12, 13]. However, LCCs are extremely reactive (e.g., cross-linking reaction and oxidation), thus it can be easily converted to $s p^{2}$ based carbon materials [14]. Moreover, there is a large difficulty in handling LCCs due to its explosiveness [14]. In these regards, two ways have been used to stabilize LCCs. One is by attaching giant molecules on the end sites [15], and the other by encapsulating LCCs in the hollow core of carbon nanotubes (CNTs) (e.g., single-, double- and multi-walled carbon nanotubes, SWCNTs, DWCNTs and MWCNTs) [16-20]. The systematic TEM and Raman studies on arc-grown MWCNTs clearly showed the presence of LCCs inside the CNTs [21]. In addition, in-situ Raman studies revealed that LCCs could be interconnecting CNTs by high pressure [22]. More interestingly, Banhart et al. reported the intrinsic electrical properties of an isolated LCC made from graphene inside a TEM, such as the metallicity under no strain, and a strain-induced transition from metal to semiconductor $[23,24]$.

It is noteworthy that no scientific discussion has been reported to-date on the possible growth mechanism of LCCs inside MWCNTs in the arc process, or how they are stabilized under high temperature thermal treatments and/or in the presence of boron atoms. Moreover, there is also no experimental study on the electrical properties of LCCs@CNT systems because of the limited filling ratio of LCCs inside CNTs, even though theoretical studies proposed that a strong coupling interaction between LCCs and CNTs would alter significantly the electronic properties of CNTs [25]. In order to establish such points experimentally, the present study attempted to grow LCCs inside MWNTs via an atmospheric arc discharge in the presence of boron atoms, and especially without using any metal catalyst. The simultaneous growth of LCCs inside MWCNTs can be explained using the close-end growth mechanism [26]. In addition, to understand the effect of the entrapped LCCs on the electrical properties of MWCNT assembly, we carried out additional boron doping and high temperature thermal annealing on as-synthesized MWCNTs and then observed the changes in the electrical conductivity before and after the disappearance of LCCs. 


\section{Experimental}

\subsection{Synthesis of a high fraction of LCCs inside MWCNTs}

MWCNTs containing LCCs were synthesized via arc discharge method using very similar experimental conditions, as described in our previous report, except of the use of boron as a chain promoter [27]. More specifically, the cathode and anode were made of graphite (or carbon) with a purity of $99.99 \%$. To precisely control the gap between the electrodes (ca. 1 $\mathrm{mm}$ ), we applied a constant current of $100 \mathrm{~A}$ (voltage $=20 \mathrm{~V}$ ). Then, by feeding argon gas containing boron trioxide (at a flow rate of $1 \mathrm{~L} / \mathrm{min}$.) into the hollow tube of the movable graphite anode (see the schematic diagram of the synthetic apparatus in Fig. S1 (a)), grey tape was deposited spirally on the outer surface of the cathode (width $=3-5 \mathrm{~mm}$, thickness $=\mathrm{ca}$. $175 \mu \mathrm{m}$ ) largely consisting of carbon nanotubes (Fig. S1 (b)). Finally, a cooling gas was used to detach the deposited thin tape from the cathode without disrupting the tape morphology.

\subsection{Boron doping and high temperature thermal treatment}

Boron doping occurred by exposing as-synthesized nanotube samples to a boron environment (10 wt. $\% \mathrm{~B}_{2} \mathrm{O}_{3}$ ) using a graphite furnace at $1400,1600,1800,2000,2200^{\circ} \mathrm{C}$ in argon. To compare with these samples, a control nanotube sample was simply heat treated at the same temperature using a graphite furnace in argon in the absence of boron.

\subsection{Structural characterizations}

Pristine and boron-doped MWCNT samples were characterized by a double Cs-corrector equipped high-resolution transmission electron microscope (Cs-HR-TEM, JEM-2100F, JEOL, acceleration voltage: $80 \mathrm{kV}$ ). Surface elemental analysis was carried out by X-ray photo-electron spectroscopy (XPS, Axis-Ultra, Shimadzu-Kratos) using a monochromatic X-ray source from an $\mathrm{Al}$ target $(12 \mathrm{kV}, 12 \mathrm{~mA})$. The degree of filling of LCCs was evaluated by Raman spectroscopy using 532, 633 and $785 \mathrm{~nm}$ laser lines under a microscope (Renishaw system).

\subsection{Electrical resistivity measurement}


The electrical resistivity and magnetoresistance measurements were carried out in the temperature range from $5 \mathrm{~K}$ to room temperature using a physical properties measurement system (PPMS, Quantum Design) in which a four-probe configuration was used by attaching the electrodes with silver paint with an inner electrode distance of approximately $1.5 \mathrm{~mm}$. The applied current was fixed at $100 \mu \mathrm{A}$ and the voltage was monitored.

\section{Results and Discussion}

The spirally deposited thin and long tape was easily detached from the rotating carbon cathode using cooling gas without observing macro-morphological changes (Fig. S1 (b)). When inspecting this tape, the nanotube purity with regard to nanoparticles was found to be ca. $80 \%$. The carbon nanotubes had an average diameter of $10.4 \mathrm{~nm}$ and an average length of $2.3 \mu \mathrm{m}$. Interestingly, a detailed TEM observation revealed that two MWCNTs with an innermost tube diameter of 0.6-0.8 nm exhibited dotted lines along the centerline (hollow core), indicating the presence of LCC (middle in Fig. 1 (a)), whereas a MWCNT with an innermost tube diameter over $1.0 \mathrm{~nm}$ exhibited encapsulated amorphous-like carbon (bottom in Fig. 1(a)), and a MWCNT with an innermost tube diameter of $0.41 \mathrm{~nm}$ did not contain any carbonaceous species (top in Fig. 1(a)). Two previous studies clearly described the importance of the innermost tube diameter (ca. $0.7 \mathrm{~nm}$ ) in the encapsulation of LCCs inside MWCNTs $[17,28]$ by considering van der Waals forces between the LCCs and the innermost tubes ( $c a$. $0.34 \mathrm{~nm}$ ). The possibility of observing the artifact such as ghost fringe in the core of a MWCNT was reported because of the interference effect and the inappropriate defocus condition [29]. To verify dotted line coming from the encased LCCs inside MWCNT, we prepared two models $((5,5) @(10,10)$ and LCC@(5,5)@(10,10)) and then obtained the simulated images using MacTempasX (an accelerating voltage $=80 \mathrm{kV}$, Cs $=5 \mu \mathrm{m}$ and defocus value $=-148 \mathrm{~nm}$ ) (Fig. S2). We found that the line profile of the TEM image we obtained ((f) in Fig. S2) is very similar to that of the simulated one obtained from LCC@(5,5)@(10,10) ((d) in Fig. S2). The image we obtained is not ghost fringe, but the actual TEM image from the encased LCC inside MWCNT. If we assume that the carbon-carbon bond length is $0.1389 \mathrm{~nm}$, the LCC exhibited a length of $20 \mathrm{~nm}$ and consisted of a chain of more than 100 carbon atoms. 
(a)
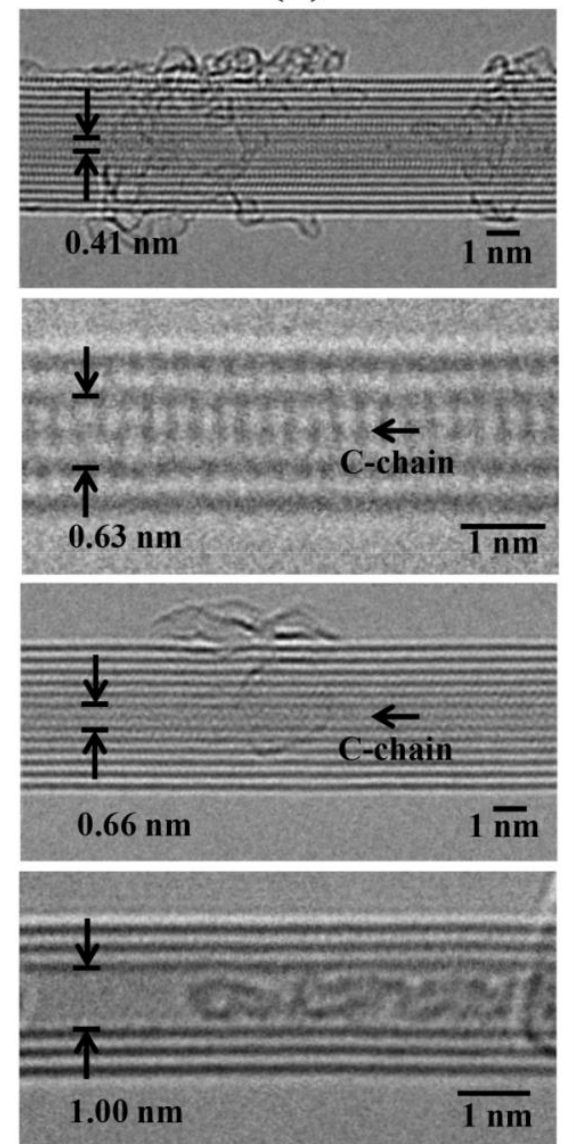

(b)

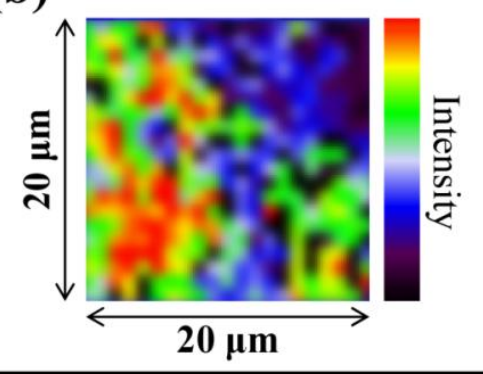

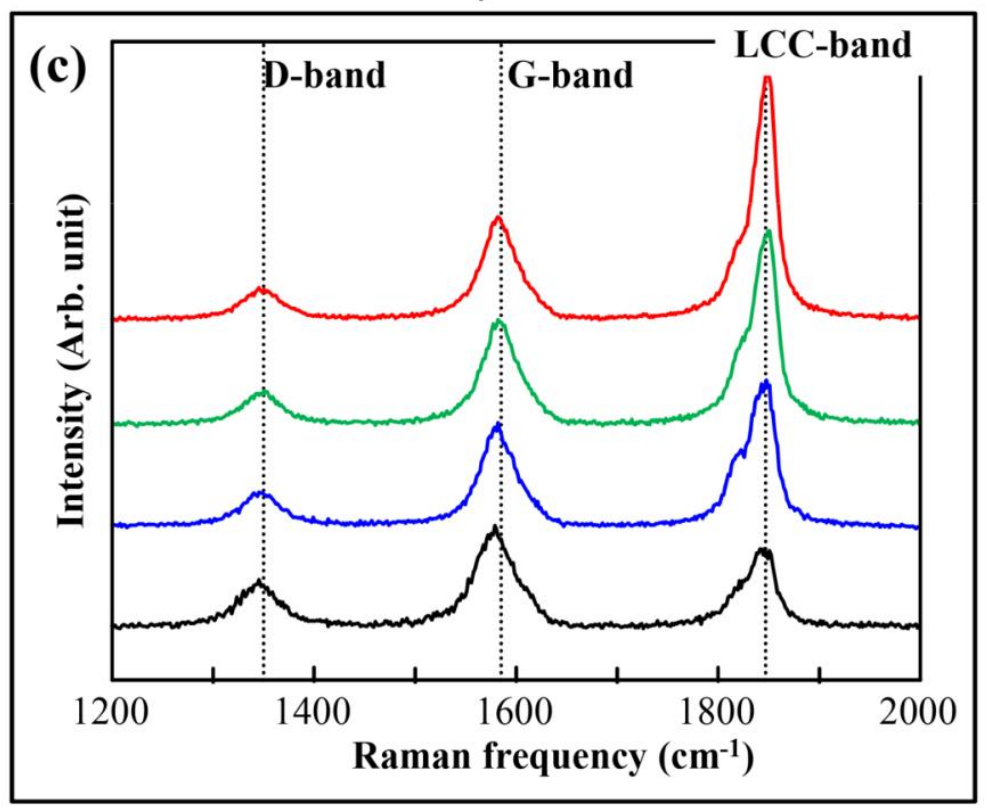

Figure 1 (a) TEM images of the pristine multi-walled carbon nanotubes synthesized via arc process. A MWCNT with an innermost tube diameter of $0.41 \mathrm{~nm}$ do not have any carbon species inside the hollow core (top), two MWCNTs with innermost tube diameters of 0.63 and $0.66 \mathrm{~nm}$ exhibited black dotted line along the centerline (middle) and a MWCNT with an innermost tube diameter of $1.0 \mathrm{~nm}$ contains amorphous like carbons (bottom). (b) Raman map image of linear carbon chains-derived bands obtained from MWCNT tape using a $532 \mathrm{~nm}$ laser line. Each color in Fig. 1 (b) exhibits its typical Raman spectrum in Fig. 1 (c). Note that we observed linear carbon chains-derived Raman peaks around $1850 \mathrm{~cm}^{-1}$ throughout the whole sample.

Unfortunately, there was no scientific discussion on the growth mechanism of LCCs within the hollow core of the innermost tube, even though experimental evidence on the presence of LCCs inside MWCNTs was reported [17, 21, 22, 28]. Initially, it was believed that a simultaneous growth of LCCs within MWCNTs is highly probable because LCCs should be perfectly encapsulated by the protective carbon layers, due to the high chemical reactivity of LCCs [14]. Therefore, open-end growth model of MWCNTs [30] is not applicable to the growth of LCCs inside MWCNTs in the arc discharge process. Based on the vapor-liquid-solid transformation, we previously suggested the closed-end growth of the 
metal-catalyst free MWCNTs in the arc process [27] by combining the model of Saito et al. [31] with the acquisition of viscous carbon clusters [32]; the overcoming accumulated charges over the surface tension of viscous carbon clusters led to ejection of carbon clusters to the cathode and the simultaneous tube elongation. Usually a round-tip morphology in arc-grown MWCNTs signifies a closed-end growth of the nanotubes. Therefore, we believe that the closed-end growth of MWCNTs is an essential condition for growing LCCs inside MWCNTs. If our suggested growth pattern is correct, super-cooling process should induce radial crystallization along the entire length of the nanotubes from the outer surface toward the internal part. In the final step, when the innermost tube diameter was in the range between 0.6 and $0.8 \mathrm{~nm}$, and the remaining carbon atoms were present inside the innermost tube, LCCs were generated and then structurally stabilized through the van der Waals interaction with innermost tubes. As mentioned in the experimental section, boron trioxide was used as nanotube growth promoter. We noted two dominant effects of boron trioxide: i) the productivity of nanotube tape largely increased up to 10 times, and ii) the frequency of observed LCCs under TEM also increased. It is also believed that the incorporation of boron atoms into carbon clusters might lead to a reduction in the surface tension, thus increasing the sustained time of the viscous state, thereby facilitating the formation of nanotubes as well as LCCs inside MWCNTs.

In previous reports, a strong Raman peak around $1850 \mathrm{~cm}^{-1}$, corresponding to a stretching mode of LCCs has been utilized to analyze the vibrational properties of the LCCs entrapped within CNTs [16-22, 33, 34]. In order to evaluate both the filling ratio and distribution of the LCCs inside MWCNTs qualitatively, we obtained Raman mapping on as-synthesized MWCNT tape using a $523 \mathrm{~nm}$ laser excitation line. The Raman map of LCCs is shown in Fig. 1 (b) and each color has its corresponding Raman spectra in Fig. 1 (c). All the Raman spectra exhibited the strongest Raman peak around $1850 \mathrm{~cm}^{-1}$, including a relatively strong G-band ( $E_{2 g 2}$ mode) at $1580 \mathrm{~cm}^{-1}$ and a weak D-band (defect induced mode) at 1350 $\mathrm{cm}^{-1}$. Such Raman mapping allowed us to conclude that the LLCs were spatially distributed homogeneously throughout the MWCNT tape with a high filling ratio. However, the intensities of the LCCs-derived peaks depended on the location of the MWCNT tape. Therefore, we obtained Raman spectra using different laser lines (Fig. 2 (a)). The 
LCCs-derived Raman peak consisted of four or five peaks, indicating the presence of LCCs of different lengths (or the number of carbon atoms). Then, we obtained the LCCs-derived Raman peaks using different laser lines and all of Raman peaks were fitted using a Lorentzian distribution function (see Fig. S3 and Table S1). We observed two distinctive Raman peaks located at $c a$. 1855 and $1825 \mathrm{~cm}^{-1}$. When plotting the integrated intensity of two LCCs-derived Raman peaks divided by the integrated intensity of the G-band as a function of excitation energy, the resonance window was clearly observed for the two types of LCCs (Fig. 2 (b)), as we reported the resonance Raman study earlier [21]. For LCC-derived Raman peak at $c a .1825 \mathrm{~cm}^{-1}$, the maximum intensity was observed when a $2.0 \mathrm{eV}$ laser line was used, whereas the maximum intensity for the LCC-derived Raman peak was observed at $1855 \mathrm{~cm}^{-1}$ with a $2.21 \mathrm{eV}$ laser line. It is theoretically expected that the Raman frequency of the LCCs decreases continuously as the number of carbon atoms increases [33, 34]. From this, it can be suggested that the LCCs we synthesized are semiconducting with the polyyne configuration (e.g., an alternating triple and single bonds) with band gaps of $2.0-2.21 \mathrm{eV}$ as a function of the LCC's length (or the number of linear carbon atoms).

(a)

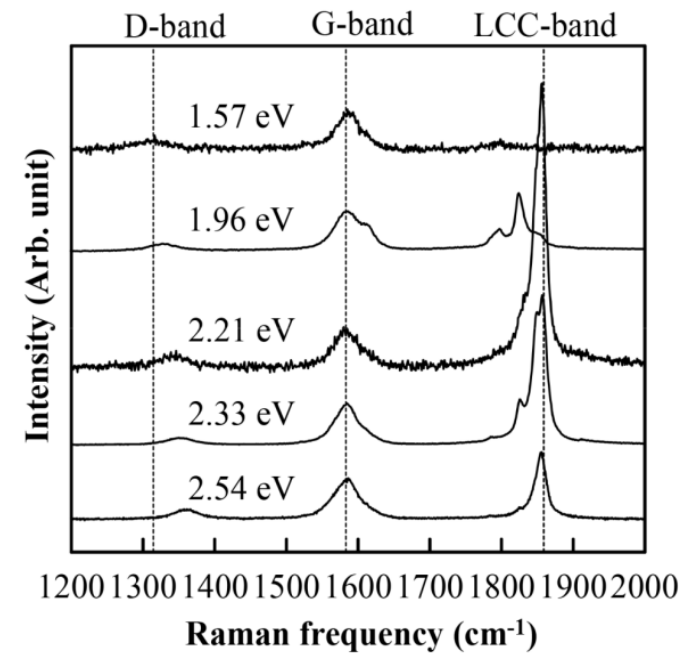

(b)

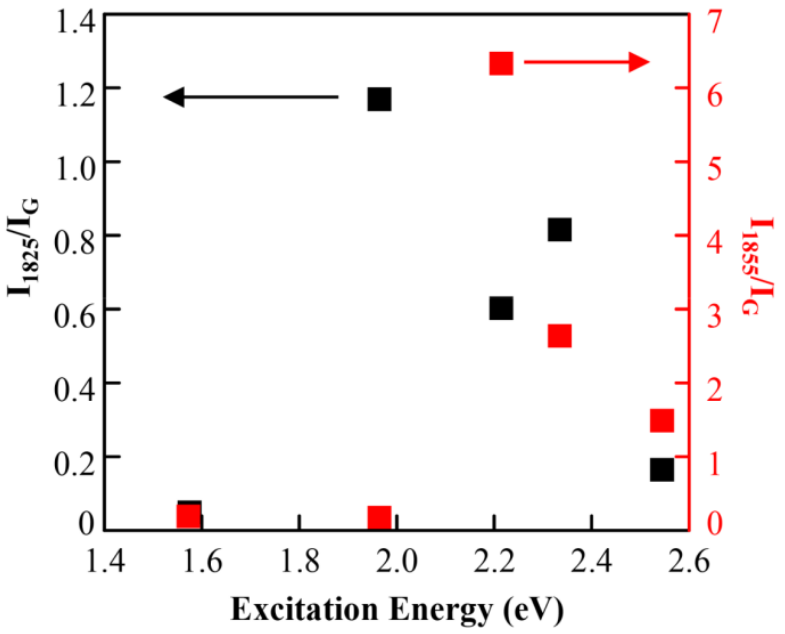

Figure 2 (a) Resonant Raman spectra of MWCNT tape using different laser lines, (b) the resonant window of two LCCs observed at 1825 and $1855 \mathrm{~cm}^{-1}$ as a function of excitation energy. Note that $I_{1825} / I_{G}$ and $I_{1855} / I_{G}$ were obtained by dividing the integrated intensity of the LCCs-derived peaks at 1825 and $1855 \mathrm{~cm}^{-1}$ by the integrated intensity of the G-band (we assumed that there was no change in the intensities of the $\mathrm{G}$ band as a function of laser line).

To understand the atomic configuration of boron atoms coming from boron trioxide within the arc process, and also to evaluate the thermal stability of the LCCs entrapped inside MWCNTs, we carried out high temperature thermal treatments as well as 
additional boron doping using a graphite furnace. Raman spectra of both thermally treated and boron doped MWCNT samples were obtained using a $532 \mathrm{~nm}$ laser line (Fig. 3 (a, b)). For the thermally treated MWCNT sample (Fig. 3 (a)), no distinctive change in both the G- and D-bands in the temperature range from 1400 to $2200^{\circ} \mathrm{C}$ was observed, indicating a high degree of crystallinity in our arc-grown MWCNTs. In other words, MWCNTs are expected to experience high thermal condition above $2200^{\circ} \mathrm{C}$ in the arc process. When looking at LCCs-derived peaks at $c a .1850 \mathrm{~cm}^{-1}$, the encapsulated LCCs inside MWCNTs were thermally stable up to $1800^{\circ} \mathrm{C}$ in argon. A clear downshift of the strong LCC-derived peaks and the newly appeared peaks located at 1820 and $1790 \mathrm{~cm}^{-1}$ could be observed when the thermal treatment temperature was increased from 1400 to $1600^{\circ} \mathrm{C}$. This can be explained by thermal energy induced coalescence between adjacent LCCs, similar to high pressure-induced coalescence of adjacent LCCs [22]. When HTT was increased further to $1800^{\circ} \mathrm{C}$, we observed only one peak located at $c a .1860 \mathrm{~cm}^{-1}$. The van der Waals interaction between the LCCs and the innermost tubes provided structural stability to the LCCs inside MWCNTs. However, the small diameter carbon nanotubes below $1 \mathrm{~nm}$ were thermally unstable when exposed to high temperature thermal treatment due to their high curvature [35-37]. The disappearance of the LCC-derived Raman peaks at lower frequency might be due to the structural destruction of the small-diameter innermost tubes induced by the high temperature thermal energy. Note that there was no peak below $500 \mathrm{~cm}^{-1}$ for Raman spectra taken with a $532 \mathrm{~nm}$ laser line. To support our assumption, Raman spectra using 633 and $785 \mathrm{~nm}$ laser lines were taken for thermally treated MWCNT samples (Fig. 3 (c) and Fig. S4). As expected, a strong radial breathing mode (RBM) above $300 \mathrm{~cm}-1$ indicates the presence of innermost tubes with a diameter of $0.7 \mathrm{~nm}$. When thermally treated above $1800^{\circ} \mathrm{C}$, the disappearance of $\mathrm{RBM}$ is a strong evidence of the thermal destruction of small diameter tubes due to a large curvature. Moreover, the low frequency Raman spectra taken with a $785 \mathrm{~nm}$ laser line clearly showed the disappearance of RBM at $280 \mathrm{~cm}^{-1}$ when HTT is increased up to $1800^{\circ} \mathrm{C}$ (Fig. S4). Thus, the LCC-derived peak we observed at high frequency for the sample prepared at $1800^{\circ} \mathrm{C}$, possibly originated from the thermally stable large-diameter innermost tubes containing LCCs.

On the other hand, for the boron doped sample, we observed largely different 
Raman behaviors (Fig. 3 (b)). For the boron doped sample heat treated at $1600^{\circ} \mathrm{C}$, the intensified D-band indicates the substitutional introduction of boron atoms into the trigonal sites of the hexagonal carbon lattice because the boron-carbon bond length is $0.5 \%$ longer than the carbon-carbon bond length [38]. With an increase in the boron doping temperature, the intensity of the D-band also increased. This indicates an increased amount of substitutional boron atoms within the MWCNT sample. However, we observed the disappearance of the LCCs-derived Raman peaks for the boron doped sample prepared at $1600^{\circ} \mathrm{C}$. A three times higher diffusion coefficient of boron atoms than carbon atoms in thermal annealing process [39] accelerated the destruction of the small diameter innermost tubes at lower temperatures.

(a)

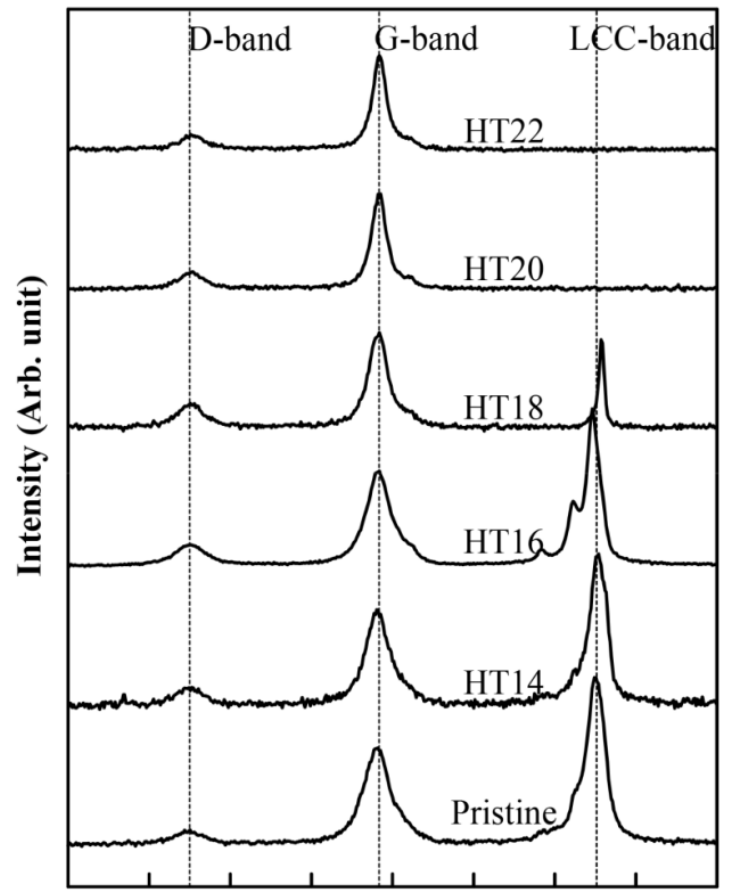

120013001400150016001700180019002000

Raman frequency $\left(\mathrm{cm}^{-1}\right)$ (b)

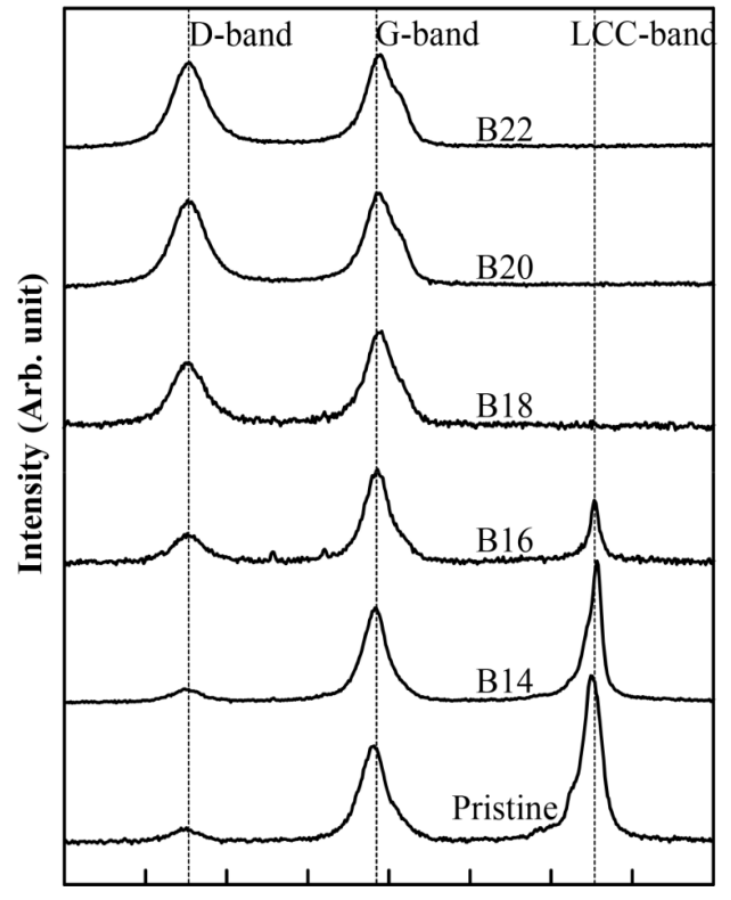

120013001400150016001700180019002000 Raman frequency $\left(\mathrm{cm}^{-1}\right)$ 


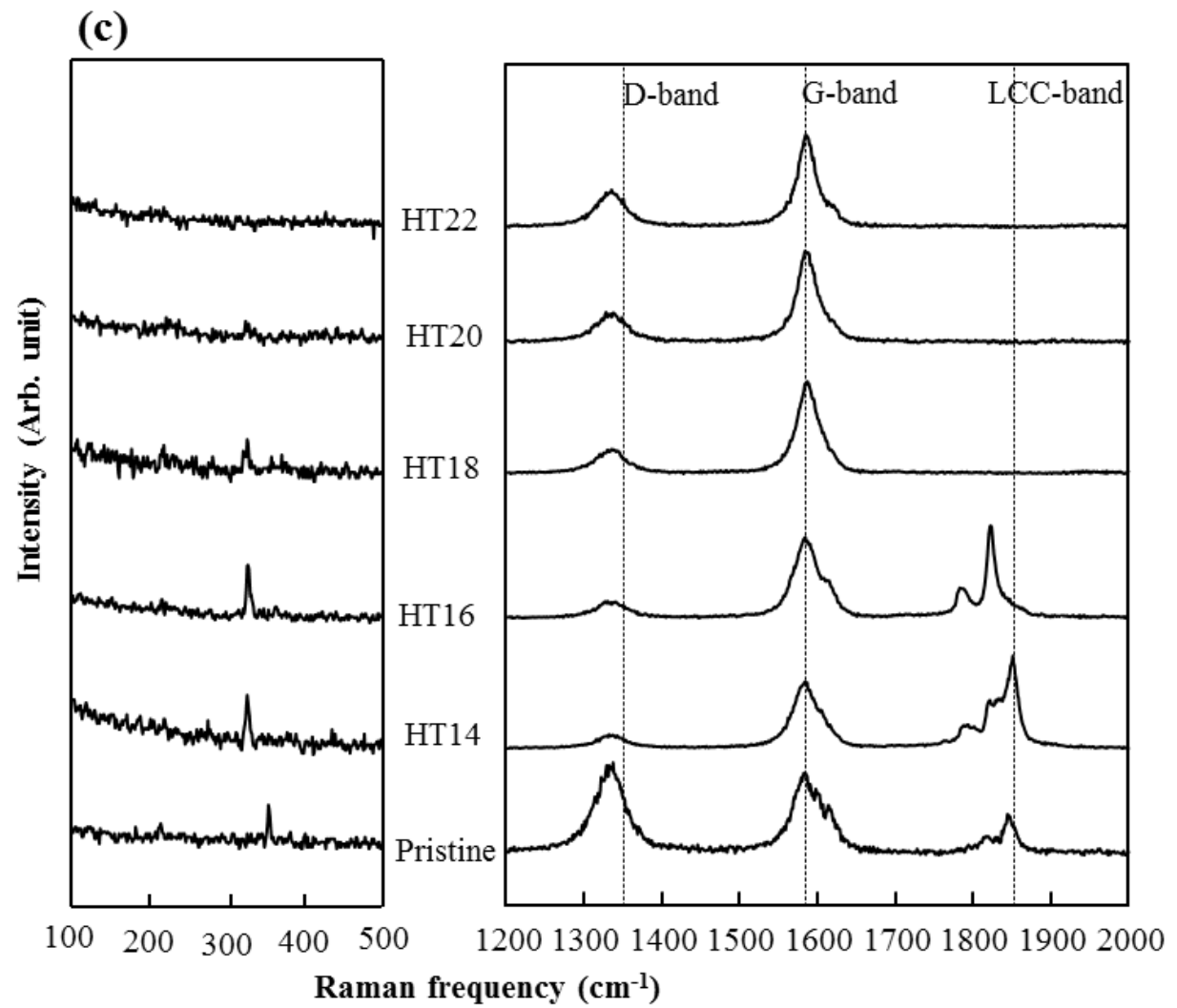

Figure 3 Raman spectra using a $532 \mathrm{~nm}$ laser line of (a) thermally treated and (b) boron doped multi-walled carbon nanotubes containing linear carbon chains that are post-treated in the temperature range from 1400 to $2200^{\circ} \mathrm{C}$ in argon. (c) Raman spectra of thermally treated multi-walled carbon nanotubes using a $633 \mathrm{~nm}$ laser line. Note that the linear carbon chains-derived Raman peak completely disappeared above $1800^{\circ} \mathrm{C}$.

Moreover, the configurations of boron atoms of both thermally treated and boron doped samples were monitored using X-ray photoelectron spectroscopy (XPS). Figure $4(a, b)$ shows B1s spectra of both thermally treated and boron doped samples prepared at the same temperature. The relatively strong peak located at $186.5 \mathrm{eV}$ was assigned to boron carbide, whereas the broad peak at ca. $187.0 \mathrm{eV}$ corresponded to the substitutional boron atoms. It was interesting to note that as-synthesized MWCNTs contained $0.4 \%$ of boron atoms in the form of oxides (e.g., $\mathrm{B}_{2} \mathrm{O}_{3}, \mathrm{BC}_{2} \mathrm{O}$ and $\mathrm{BCO}_{2}$ ). For both the samples, the amount of boron oxides decreased continuously with increasing heating temperatures, and then completely disappeared at $2000^{\circ} \mathrm{C}$, while the amount of boron clusters and the substitutional boron atoms increased as the temperature increased. Even though the B1s spectra were relatively noisy, we tried to calculate the amount of the substitutional boron atoms for both the samples (Fig. 4 (c)) because the substitutional boron atoms only affect the electronic properties of graphite, graphene and CNTs. The additional doping effect was clearly visualized by an increased 
amount of substitutional boron atoms up to 0.2 atomic \% within MWCNTs, although the amount of substitutional boron atoms of the thermally treated samples was below 0.04 atomic \%. A consecutive downshift in the binding energy of the $s p^{2}$-bonded carbon atoms (Fig. 4 (d)) obtained from the C1s spectra (see Fig. S5) supported the above-mentioned claim, indicating a lowering of the Fermi level due to formation of chemical bonds between carbon and electron-deficient boron atoms [38].

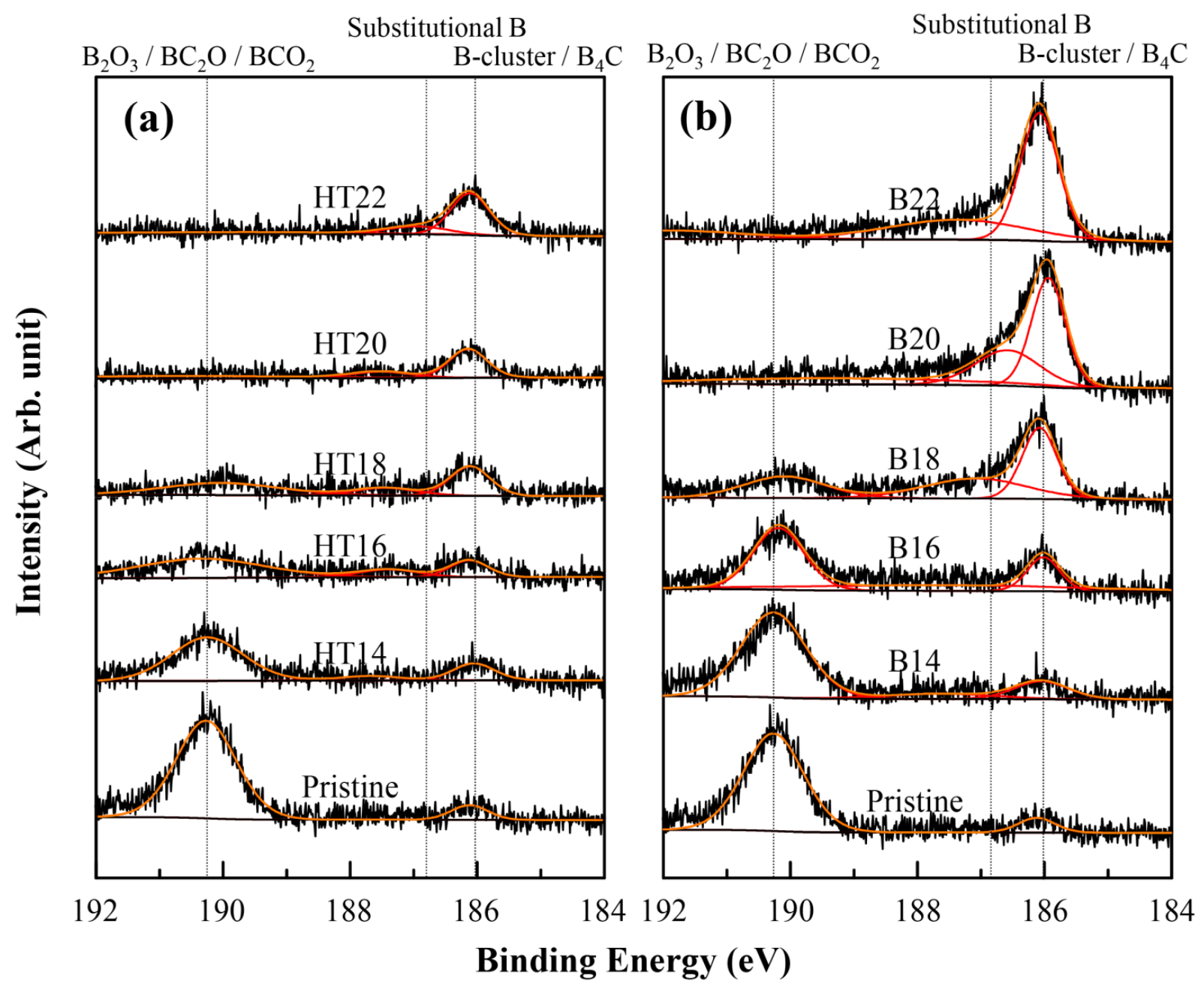


(c)

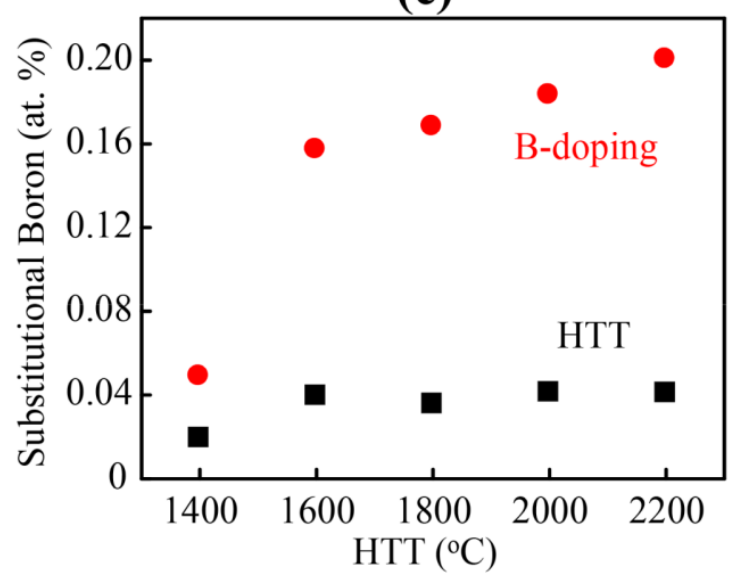

(d)

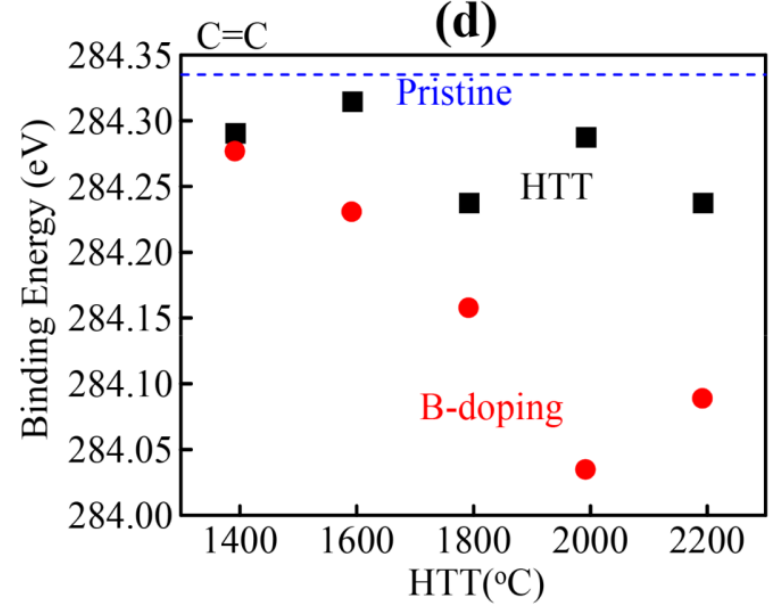

Figure 4 The B1s XPS spectra of (a) thermally treated and (b) boron doped multi-walled carbon nanotubes. (c) The substitutional amount of boron atoms as a function of thermal treatment temperature from the B1s XPS spectra (Note that the experimental error is within 0.01 atomic $\%$ based on the lowest detecting ability of the XPS apparatus) and (d) the variation of binding energy of the $s p^{2}$-bonded carbon atoms from the C1s XPS spectra (Fig. S5), where the experimental error is within $0.014 \mathrm{eV}$ (Fig. S6). The amount of substitutional boron atoms is well consistent with the downshift in the binding energy of the $s p^{2}$-bonded carbon atoms.

Finally, in order to understand the effect of the entrapped LCCs inside the MWCNTs, we measured the electrical transport properties using physical property measurement system (PPMS). As shown in Fig. 5, the boron-doped sample clearly exhibited a two times higher electrical conductivity than the thermally treated samples, due to the large increase in the hole concentration caused by $p$ doping [40]. It was generally seen that the electrical conductivities of both samples showed linearly increased behavior with increasing temperatures. However, it was also noted that the electrical conductivity of the thermally treated MWCNT sample unusually decreased, when HTT increased from 1800 to $2000^{\circ} \mathrm{C}$. As illustrated in Fig. 3 (a), Raman studies showed thermal decomposition of the LCCs inside MWCNTs within this temperature range. Therefore, this kind of unusual behavior in the electrical conductivity of the thermally treated MWCNTs can be explained by the disappearance of the LCCs inside the MWCNTs. A very similar behavior for the boron doped sample was also observed in the temperature range from 1600 to $1800^{\circ} \mathrm{C}$, in which the LCCs-derived Raman peaks disappeared. Note that the LCCs were encapsulated within the innermost tubes of the MWCNTs with three to five concentric tubules. A recent theoretical study on LCC's effect on the electronic properties of CNTs showed that the semiconducting CNT containing LCC 
displayed a metallic character due to charge transfer from the carbon nanotube to the linear carbon chain, even though both components were semiconducting [25]. In addition, we previously reported the contribution of metallic inner tubes to the electronic transport properties of DWCNT films by deactivating the outer tubes [41]. Therefore, it was expected that the metallic nature of the LCCs@innermost tubes largely contributed to the enhancement of the electrical conductivity of the MWCNT assembly.

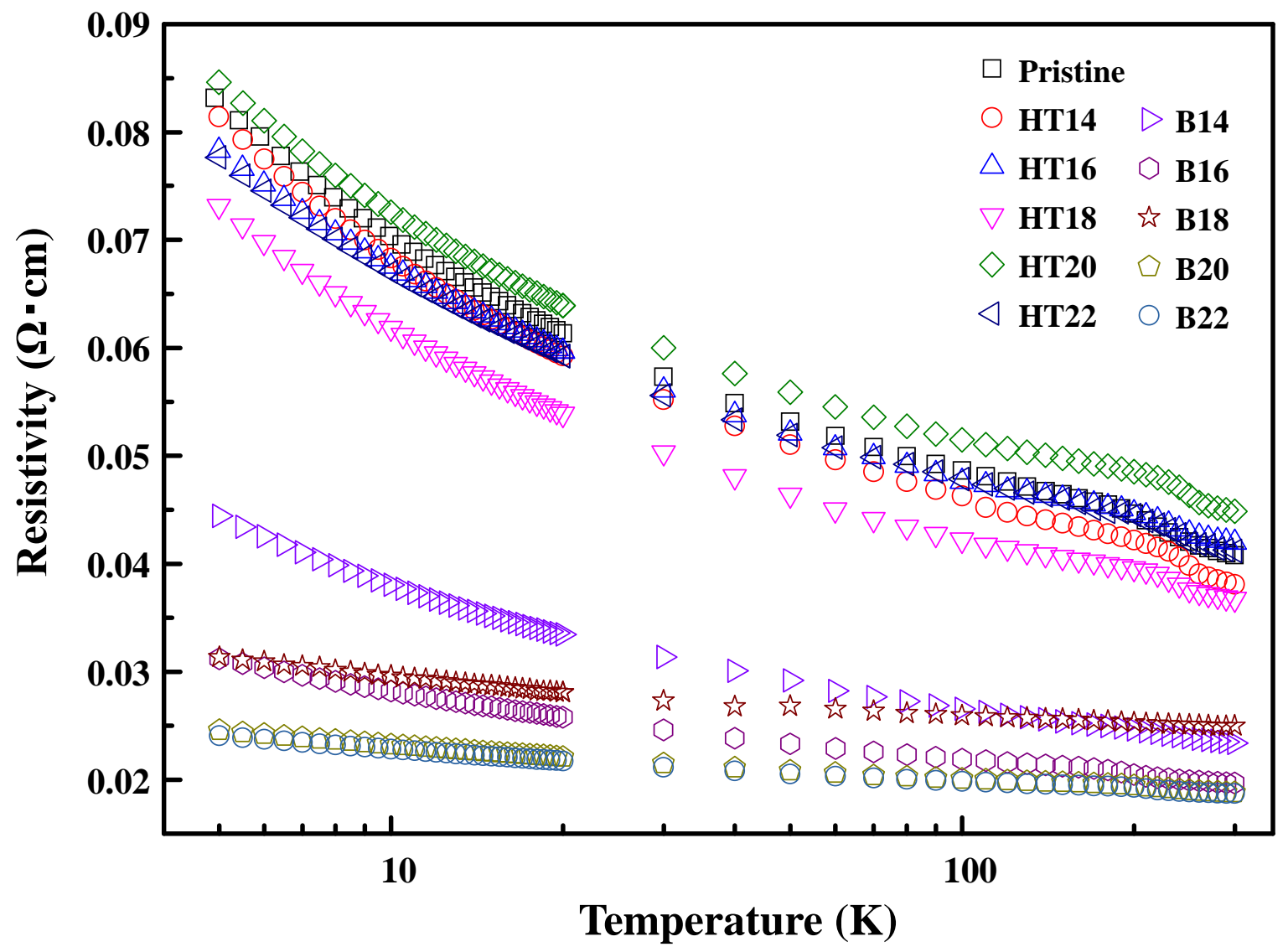

Figure 5 Temperature dependence of resistivity of the thermally treated and boron doped multi-walled carbon nanotube assemblies. Note that the resistivity of the boron doped sample is lower than the thermally treated one.

In order to understand the mechanism of electrical conduction in detail, the resistivity ratio $\left(\rho_{5 \mathrm{~K}} / \rho_{100 \mathrm{~K}}\right)$ and reduced activation energy were calculated for each sample using the following equation (Table 1).

$$
W(T)=-\frac{d \ln (\rho(T))}{d \ln (T)}=p\left(\frac{T_{0}}{T}\right)^{p} \propto T^{-p}
$$

For the thermally treated sample, there was no clear change in the resistivity and reduced activation energy at $5 \mathrm{~K}$ in comparison with those of the as-synthesized sample. However, we 
observed a metallic electronic state of boron doped sample. In order to characterize the conduction regimes more quantitatively, we calculated the $p$-value from power-law fits of $W(\mathrm{~T})$ plot and analyzed the dimensions of the variable region hopping (VRH) for each sample [42-44]. According to the VRH dimension analysis, the pristine sample showed 2D conductance behavior, but high temperature thermal annealing induced a distinctive change in the conduction behavior from 2D to 3D VRH. On the other hand, for the boron doped samples, with increasing temperature, VRH mechanism was very close to the boundary of metal-insulator transition (critical regime), thus indicating that the electronic state of boron-doped sample changed to metallic due to the formation of chemical bonds between carbon and electron-deficient boron atoms. As a result, the unusual electrical drop for the thermally treated MWCNT tape right after the disappearance of LCCs supported the strong contribution of encased LCCs to the electrical conductivity of the MWCNT assembly, although LCCs are entrapped inside the innermost tubes.

Table 1 Summary of electrical parameters calculated from the temperature dependence of resistivity of both samples.

\begin{tabular}{ccccccc}
\hline \multicolumn{2}{c}{ I.D. } & $\begin{array}{c}\rho(5 \mathrm{~K}) \\
(\mathrm{m} \Omega \cdot \mathrm{cm})\end{array}$ & $\begin{array}{c}\rho(300 \mathrm{~K}) \\
(\mathrm{m} \Omega \cdot \mathrm{cm})\end{array}$ & $\alpha^{1)}$ & $\mathrm{W}(1.9 \mathrm{~K})^{2)}$ & $p^{3)}$ \\
\hline \multirow{6}{*}{ Pristine } & 83.2 & 40.8 & 1.71 & 0.26 & 0.27 \\
\hline \multirow{6}{*}{ Thermal } & $1400^{\circ} \mathrm{C}$ & 81.4 & 38.1 & 1.76 & 0.27 & 0.28 \\
& $1600^{\circ} \mathrm{C}$ & 78.3 & 42.0 & 1.64 & 0.23 & 0.26 \\
treatment & $1800^{\circ} \mathrm{C}$ & 76.2 & $36.7 \pm 1.35^{4)}$ & 1.73 & 0.26 & 0.28 \\
& $2000^{\circ} \mathrm{C}$ & 89.6 & $43.8 \pm 1.16^{5)}$ & 1.64 & 0.24 & 0.26 \\
& $2200^{\circ} \mathrm{C}$ & 77.6 & 41.2 & 1.62 & 0.23 & 0.24 \\
\hline \multirow{6}{*}{ Boron } & $1400^{\circ} \mathrm{C}$ & 44.4 & 23.4 & 1.67 & 0.24 & 0.27 \\
& $1600^{\circ} \mathrm{C}$ & 31.2 & 19.7 & 1.42 & 0.15 & 0.19 \\
doping & $1800^{\circ} \mathrm{C}$ & 31.4 & 25.0 & 1.21 & 0.087 & 0.15 \\
& $2000^{\circ} \mathrm{C}$ & 24.6 & 18.8 & 1.22 & 0.082 & 0.089 \\
& $2200^{\circ} \mathrm{C}$ & 24.1 & 18.7 & 1.21 & 0.081 & 0.093 \\
\hline
\end{tabular}

${ }^{1)} \alpha(\rho(5 \mathrm{~K}) / \rho(100 \mathrm{~K}))$ indicates resistivity ratio, ${ }^{2)} \mathrm{W}$ indicates reduced activation energy and ${ }^{3)}$ 
$p$ is the dimension of the variable range hopping. ${ }^{4)}$ To secure the reproducibility of the electrical resistivity data, 4 different electrodes from the thermally treated MWCNT samples at 1800 (see Fig. S7) and $2000^{\circ} \mathrm{C}$ were prepared and then their temperature dependent resistivity was measured using the physical property measurement system. Then, the average value and the standard deviations are included (see Table S2).

\section{Conclusion}

We successfully synthesized metal-free MWCNTs containing a large portion of LCCs via atmospheric arc method using boron trioxide as the promoter during nanotube synthesis. The closed-end growth mechanism of the MWCNTs in the arc process was more suitable for growing chemically active LCCs inside MWCNTs. More specifically, the overcoming accumulated charges over the surface tension of viscous carbon cluster led to the ejection of carbon clusters to the cathode and simultaneously caused the tube elongation and growth. The subsequent cooling process induced radial crystallization along the entire length of the MWCNTs from the outer surface toward the internal part. Eventually, when the innermost tube diameter was in the range of $0.6-0.8 \mathrm{~nm}$ and the residual carbon atoms were present inside the innermost tube, LCCs were generated. The structural stability of the LCCs inside MWCNTs were closely related with the structural stability of the innermost tubes (or host materials), when they were exposed to high temperature thermal annealing or boron doping. This is because they were energetically stabilized within innermost tubes through van der Waals interactions. The unusual decrease in the electrical conductivity of MWCNTs in the temperature range from 1800 to $2000^{\circ} \mathrm{C}$ in argon was a strong experimental evidence of the significant contribution of the LCCs to the electrical conductivity of the MWCNT assembly, even though the LCCs were present in the hollow core of the innermost tubes. If we are able to incorporate a high portion of LCCs inside SWCNTs in a controllable manner, all metallic nanotube film with extremely high electrical conductivity could be synthesized.

\section{Acknowledgements}

Y.A.K. acknowledges the financial support from the National Research Foundation of Korea (NRF) grant funded by the Korea government (MSIP) (No. NRF-2014R1A2A1A10050585) 
and from the framework of international cooperation program managed by the National Research Foundation of Korea (NRF-2015K2A2A4000110, FY2015). 


\section{References}

[1] Januszewski JA, Tykwinski RR. Synthesis and properties of long [n] cumulenes ( $\mathrm{n}>5$ ). Chem Soc Rev 2014; 43:3184-203.

[2] Cataldo F. Polyynes: Synthesis, Properties, and Applications. New York: CRC Press; 2006.

[3] Liu M, Artyukhov VI, Lee HK, Xu F, Yakobson BI. Carbyne from first principles: Chain of C Atoms, a nanorod or a nanorope. ACS Nano 2013; 7:10075-82.

[4] Zhang Y, Su Y, Wang L, Kong ESW, Chen XS, Zhang Y. A one-dimensional extremely covalent material: monatomic carbon linear chain. Nanoscale Res Lett 2011; 6:577.

[5] Tobe Y, Wakabayashi T. Carbon-rich compounds: acetylene-based carbon allotropes. In: Diederich F, Stang PJ, Tykwinski RR, editors. Acetylene chemistry, chemistry, biology and material science, Weinheim: WILEY-VCH Verlag GmbH \& Co. KGaA; 2005, p. $387-426$.

[6] Slepkov AD, Hegmann FA, Eisler S, Elliott E, Tykwinski RR. The surprising nonlinear optical properties of conjugated polyyne oligomers. J Chem Phys 2004; 120:6807.

[7] Shiraishi S, Kobayashi T, Oya A. Electrochemical lithium ion doping \& undoping behavior of carbyne-like carbon film electrode. Chem Lett 2005; 34:1678-9.

[8] Heimann RB, Evsyukov SE, Kavan L. Carbyne and Carbynoid Structures. Dordrech: Kluwer Academic Publishers; 1999.

[9] Jin C, Lan H, Peng L, Suenaga K, Iijima S. Deriving carbon atomic chains from graphene. Phys Rev Lett 2009; 102:205501.

[10] Chuvillin A, Meyer JC, Algara-Siller G, Kaiser U. From graphene constrictions to single carbon chains. New J Phys 2009; 11:083019.

[11] Casillas G, Mayoral A, Liu M, Ponce A, Artyukhov VI, Yakobson BI, et al., New insights into the properties and interactions of carbon chains as revealed by HRTEM and DFT analysis. Carbon 2014; 66:436-41.

[12] Tsuji M, Tsuji T, Kuboyama S, Yoon S.-H, Korai Y, Tsujimoto T, et al. Formation of hydrogen-capped polyynes by laser ablation of graphite particles suspended in solution. Chem Phys Lett 2002; 355:101-8.

[13] Cataldo F. Synthesis of polyynes in a submerged electric arc in organic solvents. Carbon 
2004; 42:129-142.

[14] Baughman RH. Dangerously seeking linear carbon. Science 2006;312:1009-10.

[15] Gibtner T, Hampel F, Gisselbrecht J-P, Hirsch A. End-cap stabilized oligoynes: Model compounds for the linear sp carbon allotrope carbyne. Chem Eur J 2002;68:408-32.

[16] Nishide D, Dohi H, Wakabayashi T, Nishibori E, Aoyagi S, Ishida M, et al. Single-wall carbon nanotubes encaging linear chain $\mathrm{C} 10 \mathrm{H} 2$ polyyne molecules inside. Chem Phys Lett 2006;428:356-60.

[17] Zhao X, Ando Y, Liu Y, Jinno M, Suzuki T. Carbon nanowire made of a long linear carbon chain inserted inside a multiwalled carbon nanotube. Phys Rev Lett 2003;90:187401.

[18] Zhao C, Kitaura R, Hara H, Irele S, Shinohara H. Growth of linear carbon chains inside thin double-walled carbon nanotubes. J Phys Chem C 2011;115:13166-70.

[19] Shi L, Sheng L, Yu L, An K, Ando Y, Zhao X. Ultra-thin double-walled carbon nanotubes: A novel nanocontainer for preparing atomic wires. Nano Res 2011;4:759-66.

[20] Endo M, Kim YA, Hayashi T, Muramatsu H, Terrones M, Saito R, et al. Nanotube coalescence-inducing mode: A novel vibrational mode in carbon systems. Small 2006;2:1031-6.

[21] Andrade NF, Vasconcelos TL, Gouvea CP, Archanjo BS, Achete CA, Kim YA, et al. Linear carbon chains encapsulated in multiwall carbon nanotubes: Resonance Raman spectroscopy and transmission electron microscopy studies. Carbon 2015;90:172-80.

[22] Andrade NF, Aguiar AL, Kim Y A, Endo M, Freire PTC, Brunetto G, et al. Linear carbon chains under high-pressure conditions. J Phys Chem C 2015;119:10669-76.

[23] Cretu O, Botello-Mendez AR, Janowska I, Pham-Huu C, Charlier J-C, Banhart F. Electrical transport measured in atomic carbon chains. Nano Lett 2013;13:3487-93.

[24] La Torre A, Botello-Mendez A, Baaziz W, Charlier J-C, Banhart F Strain-induced metal-semiconducting transition observed in atomic carbon chains. Nature Commun $2015 ; 6: 6636$.

[25] Tapia A, Aguilera L, Cab C, Medina-Esquivel RA, de Coss R, Canto G. Density functional study of the metallization of a linear carbon chain inside single wall carbon nanotubes. Carbon 2010;48:4057-62. 
[26] Endo M, Kroto HW. Formation of carbon nanofibers. J Phys Chem 1992;96:6941-4.

[27] Kim YA, Muramatsu H, Hayashi T, Endo M. Catalytic metal-free formation of multi-walled carbon nanotubes in atmospheric arc discharge. Carbon 2012;50:4588-95.

[28] Wang Z, Ke X, Zhu Z, Zhang F, Ruan M, Yang J. Carbon-atom chain formation in the core of nanotubes. Phys Rev B 2000;61:R2472-4.

[29] Hayashi T, Muramatsu H, Kim YA, Kajitani H, Imai S, Kawakami H, et al. TEM image simulation study of small carbon nanotubes and carbon nanowire. Carbon 2006;44:1130-6.

[30] Iijima S, Ajayan PM, Ichihashi T. Growth model for carbon nanotubes. Phys Rev Lett 1992;69:3100-3.

[31] Saito Y, Yoshikawa T, Inagaki M, Tomita M, Hayashi T. Growth and structure of graphitic tubules and polyhedral particles in arc-discharge. Chem Phys Lett 1993;204:277-82.

[32] de Heer WA, Poncharal P, Berger C, Gezo J, Song Z, Bettini J, et al. Liquid carbon, carbon-glass beads, and the crystallization of carbon nanotubes. Science 2005;307:907-10.

[33] Milani A, Tommasini M, Russo V, Li Bassi A, Lucotti A, Cataldo F, et al. Raman spectroscopy as a tool to investigate the structure and electronic properties of carbon-atom wires. Beilstein J Nanotechnol 2015;6:480-91.

[34] Haque MM, Yin L, Nugraha ART, Saito R. Vibrational and NMR properties of polyynes. Carbon 2011;49:3340-5.

[35] Metenier K, Bonnamy S, Beguin F, Journet C, Bernier P, Lamy de La Chapelle M, et al. Coalescence of single-walled carbon nanotubes and formation of multi-walled carbon nanotubes under high-temperature treatments. Carbon 2002;40:1765-73.

[36] Yudasaka M, Ichihashi T, Kasuya D, Kataura H, Iijima S. Structure changes of single-wall carbon nanotubes and single-wall carbon nanohorns caused by heat treatment. Carbon 2003;41:1273-80.

[37] Kim YA, Muramatsu H, Hayashi T, Endo M, Terrones M, Dresselhaus MS. Thermal stability and structural changes of double-walled carbon nanotubes by heat treatment. Chem Phys Lett 2004;398:87-92. 
[38] Kim YA, Fujisawa K, Muramatsu H, Hayashi T, Endo M, Fujimori T, et al. Raman spectroscopy of boron-doped single-layer graphene. ACS Nano 2012;6:6293-300.

[39] Thrower PA, Mayer RM. Point defects and self-diffusion in graphite. Physica Status Solidi (a) 1978;47:11-37.

[40] Kim YA, Aoki S, Fujisawa K, Ko Y-I, Yang K-S, Yang C-M, et al. Defect-assisted heavily and substitutionally boron-doped thin multiwalled carbon nanotubes using high-temperature thermal diffusion. J Phys Chem C 2014;118:4454-9.

[41] Fujisawa K, Komiyama K, Muramatsu H, Shimamoto D, Tojo T, Kim YA, et al. Chirality-dependent transport in double-walled carbon nanotube assemblies: The role of inner tubes. ACS Nano 2011;5:7547-54.

[42] Menon R, Yoon CO, Moses D, Heeger AJ, Cao Y. Transport in polyaniline near the critical regime of the metal-insulator transition. Phys Rev B 1993;48:17685.

[43] Vavro J, Kikkawa JM, Fischer JE. Metal-insulator transition in doped single-wall carbon nanotubes. Phys Rev B 2005;71:155410.

[44] Vora PM, Gopu P, Rosario-Canales M, Perez CR, Gogotsi Y, Santiago-Aviles JJ, et al. Correlating magnetotransport and diamagnetism of $s p^{2}$-bonded carbon networks through the metal-insulator transition. Phys Rev B 2011;84:155114. 